\title{
ON FUNCTIONS AND EQUATIONS IN DISTRIBUTIVE LATTICES
}

\author{
by SERGIU RUDEANU
}

(Received 15th July 1967)

Summary. In [1], R. L. Goodstein has extended some well-known theorems on functions and equations in a Boolean algebra to the case of a distributive lattice $L$ with 0 and 1 . The purpose of this paper is to prove that most of Goodstein's theorems, as well as some additional results, are still valid in the case when $L$ is not required to have least and greatest elements.

Throughout this paper, we shall always assume that $\langle L, \cup,$.$\rangle is a distributive$ lattice.

The definition of a lattice function of $n$ variables is as follows:

1. The elements $a, b, c, \ldots, A, B, C, \ldots$ of $L$ are lattice functions.

2. The functions $\varepsilon_{i}$, defined by

$$
\varepsilon_{i}\left(x_{1}, \ldots, x_{n}\right)=x_{i} \quad \forall x_{1}, \ldots, x_{n} \in L \quad(i=1,2, \ldots, n)
$$

are lattice functions.

3. If $f, g: L^{n} \rightarrow L$ are lattice functions, then the functions $f \cup g$ and $f g$, defined by

$$
\begin{gathered}
(f \cup g)\left(x_{1}, \ldots, x_{n}\right)=f\left(x_{1}, \ldots, x_{n}\right) \cup g\left(x_{1}, \ldots, x_{n}\right) \forall x_{1}, \ldots, x_{n} \in L, \\
(f g)\left(x_{1}, \ldots, x_{n}\right)=f\left(x_{1}, \ldots, x_{n}\right) g\left(x_{1}, \ldots, x_{n}\right) \forall x_{1}, \ldots, x_{n} \in L,
\end{gathered}
$$

are lattice functions.

Lemma 1. The inequality

$$
a \cup b x \leqq c \cup d x
$$

is equivalent to the following system of inequalities:

$$
\begin{gathered}
a \leqq c \cup d, \\
b x \leqq c \cup d, \\
a \leqq c \cup x .
\end{gathered}
$$

Proof. Since $c \cup d x=(c \cup d)(c \cup x)$, the inequality (4) is equivalent to the system consisting of (5), (6), (7) and $b x \leqq c \cup x$; but the last inequality is identically satisfied.

$$
\text { E.M.S.-D }
$$


Lemma 2. The inequality (4) is identically satisfied if and only if

and

$$
a \leqq c
$$

$$
b \leqq c \cup d .
$$

Proof. If (4) is identically satisfied so are the relations (6) and (7). Taking $x=b$ in (6) and $x=c$ in (7), we get (9) and (8), respectively. Conversely, the relations (8) and (9) imply that $a \cup b x \leqq c \cup(c \cup d) x=c \cup d x$ for all $x \in L$.

Lemma 3. The equation

$$
a \cup b x=c \cup d x
$$

is identically satisfied if and only if

and

$$
a=c
$$

$$
a \cup b=c \cup d .
$$

Proof. The identity (10) holds if and only if $a \leqq c, b \leqq c \cup d, c \leqq a$, $d \leqq a \cup b$, by Lemma 2. These inequalities imply, in turn, $a=c$ and

$$
a \cup b=c \cup b \leqq c \cup d=a \cup d \leqq a \cup b .
$$

Conversely, (11) and (12) imply

$$
a \cup b x=a \cup(a \cup b) x=c \cup(c \cup d) x=c \cup d x .
$$

We come now to the study of lattice functions. It was proved in [1] that every lattice function can be written in the form $f(x)=A \cup B x$, where $A \leqq B$. Let $g(x)=C \cup D x$, where $C \leqq D$, be another lattice function. Lemma 2 shows that the inequality $f(x) \leqq g(x)$ is identically satisfied if and only if $A \leqq C$ and $B \leqq D$. Hence $f=g$ if and only if $A=C$ and $B=D$.

For every $n+1$ elements $a, x_{1}, \ldots, x_{n} \in L$ and for every $n$ indices $\alpha_{1}, \ldots, \alpha_{n}$ equal to 0 or 1 , let us put

$$
a x_{1}^{\alpha_{1}} \ldots x_{n}^{\alpha_{n}}=\left\{\begin{array}{l}
a x_{i_{1}} \ldots x_{i_{m}} \text { if } \alpha_{i_{1}}=\ldots=\alpha_{i_{m}}=1, \text { the other } \alpha_{j}=0 \\
a, \text { if all } \alpha_{j}=0 .
\end{array}\right.
$$

The above results can be generalized as follows.

Theorem 1. Every lattice function $f: L^{n} \rightarrow L$ can uniquely be written in the canonical form

$$
f\left(x_{1}, \ldots, x_{n}\right)=\bigcup_{i_{1}}, \ldots, i_{n} F\left(i_{1}, \ldots, i_{n}\right) x_{1}^{i_{1}} \ldots x_{n}^{i_{n}},
$$

where $F\left(i_{1}, \ldots, i_{n}\right)$ are elements of $L$ such that

$$
i_{1} \leqq j_{1}, \ldots, i_{n} \leqq j_{n} \text { imply } F\left(i_{1}, \ldots, i_{n}\right) \leqq F\left(j_{1}, \ldots, j_{n}\right) \text {. }
$$

Theorem 2. Let (14) and

$$
g\left(x_{1}, \ldots, x_{n}\right)=\bigcup_{i_{1}}, \ldots, i_{n} G\left(i_{1}, \ldots, i_{n}\right) x_{1}^{i_{1}} \ldots x_{n}^{i_{n}}
$$

be the canonical forms of the functions $f$ and $g$. The inequality $f \leqq g$, that is

$$
f\left(x_{1}, \ldots, x_{n}\right) \leqq g\left(x_{1}, \ldots, x_{n}\right) \quad \forall x_{1}, \ldots, x_{n} \in L
$$


holds if and only if

$$
F\left(i_{1}, \ldots, i_{n}\right) \leqq G\left(i_{1}, \ldots, i_{n}\right) \quad \forall i_{1}, \ldots, i_{n} \in\{0,1\} .
$$

Proof of Theorems 1 and 2. For $n=1$, the theorems were proved before. The next step of the inductive proof is carried out as follows.

The function $f$ can be written in the form

$$
f\left(x_{1}, \ldots, x_{n}\right)=f^{\prime}\left(x_{1}, \ldots, x_{n-1}\right) \cup f^{\prime \prime}\left(x_{1}, \ldots, x_{n-1}\right) x_{n},
$$

where $f^{\prime}$ and $f^{\prime \prime}$ are lattice functions satisfying the identity

$$
f^{\prime}\left(x_{1}, \ldots, x_{n-1}\right) \leqq f^{\prime \prime}\left(x_{1}, \ldots, x_{n-1}\right) .
$$

In view of the inductive hypothesis, we have

$$
F^{\prime}\left(i_{1}, \ldots, i_{n-1}\right) \leqq F^{\prime \prime}\left(i_{1}, \ldots, i_{n-1}\right) \quad \forall i_{1}, \ldots, i_{n-1} \in\{0,1\},
$$

where $F^{\prime}$ and $F^{\prime \prime}$ are the coefficients of the canonical forms of the functions $f^{\prime}$ and $f^{\prime \prime}$, respectively.

It follows that the function $f$ can be written in the form (14), with

and

$$
F\left(i_{1}, \ldots, i_{n-1}, 0\right)=F^{\prime}\left(i_{1}, \ldots, i_{n-1}\right)
$$

$$
F\left(i_{1}, \ldots, i_{n-1}, 1\right)=F^{\prime \prime}\left(i_{1}, \ldots, i_{n-1}\right) \text {. }
$$

By the inductive hypothesis, both $F^{\prime}$ and $F^{\prime \prime}$ have the property (15); taking into account (21), we see that the constants $F$ have the property (15) too.

Furthermore, let $g\left(x_{1}, \ldots, x_{n}\right)=g^{\prime}\left(x_{1}, \ldots, x_{n-1}\right) \cup g^{\prime \prime}\left(x_{1}, \ldots, x_{n-1}\right) x_{n}$, where $g^{\prime} \leqq g^{\prime \prime}$, be another lattice function. The inequality $f \leqq g$ holds identically if and only if the inequalities $f^{\prime} \leqq g^{\prime}$ and $f^{\prime \prime} \leqq g^{\prime \prime}$ hold identically, i.e. if and only if

$$
F^{\prime}\left(i_{1}, \ldots, i_{n-1}\right) \leqq G^{\prime}\left(i_{1}, \ldots, i_{n-1}\right) \text { and } F^{\prime \prime}\left(i_{1}, \ldots, i_{n-1}\right) \leqq G^{\prime \prime}\left(i_{1}, \ldots, i_{n-1}\right)
$$

$\forall i_{1}, \ldots, i_{n-1} \in\{0,1\}$. This means that the relation (17) is equivalent to (18).

Hence we deduce the uniqueness of the representation (14), which we state separately, thus completing the proof:

Corollary 1. The identity $f=g$ holds if and only if

$$
F\left(i_{1}, \ldots, i_{n}\right)=G\left(i_{1}, \ldots, i_{n}\right) \quad \forall i_{1}, \ldots, i_{n} \in\{0,1\},
$$

where $F$ and $G$ are the coefficients occurring in the canonical forms of the functions $f$ and $g$, respectively.

Theorem 2 and Corollary 1 generalize the so-called "verification theorem" due to Löwenheim [4]. Theorem 1 and Theorem 3 below are also generalizations of a well-known result on Boolean functions.

Let us now determine the canonical forms of the functions $f \cup g$ and $f g$, defined by (2) and (3), respectively.

Theorem 3. Let (14) and (16) be the canonical forms of the functions $f$ and $g$, respectively. Then

$$
(f \cup g)\left(x_{1}, \ldots, x_{n}\right)=\bigcup_{i_{1}, \ldots, i_{n}}\left[F\left(i_{1}, \ldots, i_{n}\right) \cup G\left(i_{1}, \ldots, i_{n}\right)\right] x_{1}^{i_{1}} \ldots x_{n}^{i_{n}}
$$


and

$$
(f g)\left(x_{1}, \ldots, x_{n}\right)=\bigcup_{i_{1}, \ldots, i_{n}} F\left(i_{1}, \ldots, i_{n}\right) G\left(i_{1}, \ldots, i_{n}\right) x_{1}^{i_{1}} \ldots x_{n}^{i_{n}}
$$

are the canonical forms of the functions $f \cup g$ and $f g$, respectively.

Proof. Relation (23) results immediately from (14) and (16). Since $a x^{i} x^{j}=a x^{i \cup j}$, it follows also that

$$
(f g)\left(x_{1}, \ldots, x_{n}\right)=\bigcup_{i_{1}, \ldots, i_{n}}\left[\bigcup_{j \cup k=i} F\left(j_{1}, \ldots, j_{n}\right) G\left(k_{1}, \ldots, k_{n}\right)\right] x_{1}^{i_{1}} \ldots x_{n}^{i_{n}},
$$

where $\bigcup_{j \cup k=i}$ means that the join is extended over those indices

$$
j_{1}, \ldots, j_{n}, i_{1}, \ldots, i_{n} \in\{0,1\} \text { which satisfy } j_{1} \cup k_{1}=i_{1}, \ldots, j_{n} \cup k_{n}=i_{n} .
$$

Since both $F$ and $G$ have the property (15), it follows that

$$
\bigcup_{j \cup k} F\left(j_{1}, \ldots, j_{n}\right) G\left(k_{1}, \ldots, k_{n}\right)=F\left(i_{1}, \ldots, i_{n}\right) G\left(i_{1}, \ldots, i_{n}\right)
$$

and hence (25) reduces to (24).

Since the constants $F \cup G$, as well as the constants $F G$, have obviously the property (15), it follows that (23) and (24) are actually the canonical forms of $f \cup g$ and $f g$, respectively.

The above theorems can be applied to the study of lattice equations.

As was remarked in [1], any equation $A=B$ is equivalent to the inequality $A \cup B \leqq A B$. Hence we shall focus our attention on inequalities of the form

$$
f\left(x_{1}, \ldots, x_{n}\right) \leqq g\left(x_{1}, \ldots, x_{n}\right)
$$

We begin with the following

Lemma 4. The inequality

$$
f(x) \leqq g(x)
$$

is solvable if and only if the relation

$$
F(0) \leqq G(1)
$$

holds. If this condition is fulfilled, then an element $x \in L$ is a solution of (28) if and only if

and

$$
F(1) x \leqq G(1)
$$

$$
F(0) \leqq G(0) \cup x .
$$

Proof. The result follows immediately from Lemma 1 and Theorem 1.

Corollary 2. If $x^{\prime}$ and $x^{\prime \prime}$ satisfy the inequality (28) and $x \in\left[x^{\prime}, x^{\prime \prime}\right]$, i.e.

$$
x^{\prime} x^{\prime \prime} \leqq x \leqq x^{\prime} \cup x^{\prime \prime},
$$

then $x$ is also a solution of (28).

Proof. It follows from Lemma 4 that $F(1) x^{\prime} \leqq G(1)$ and $F(1) x^{\prime \prime} \leqq G(1)$; hence $F(1) x \leqq F(1)\left(x^{\prime} \cup x^{\prime \prime}\right) \leqq G(1)$. The inequality (31) is proved similarly. Therefore $x$ satisfies (28), again by Lemma 4 . 
Let us now associate with each lattice function $f\left(x_{1}, \ldots, x_{n}\right)$, the lattice functions

$$
F\left(x_{1}, \ldots, x_{m} ; i_{m+1}, \ldots, i_{n}\right)=\bigcup_{i_{1}, \ldots, i_{m}} F\left(i_{1}, \ldots, i_{m}, i_{m+1}, \ldots, i_{n}\right) x_{1}^{i_{1}} \ldots x_{m}^{i} .
$$

Theorem 4. The inequality (27) is solvable if and only if relation

$$
F(0, \ldots, 0) \leqq G(1, \ldots, 1)
$$

holds. If this condition is fulfilled, then a vector $\left(x_{1}, \ldots, x_{n}\right) \in L^{n}$ is a solution of (27) if and only if it satisfies the relations

and

$$
F\left(x_{1}, \ldots, x_{k-1}, 0,0, \ldots, 0\right) \leqq G\left(x_{1}, \ldots, x_{k-1}, 0,1, \ldots, 1\right) \cup x_{k}
$$

$$
F\left(x_{1}, \ldots, x_{k-1}, 1,0, \ldots, 0\right) x_{k} \leqq G\left(x_{1}, \ldots, x_{k-1}, 1,1, \ldots, 1\right)
$$

for $k=1,2, \ldots, n$.

Proof. For $n=1$, Theorem 4 reduces to Lemma 4. The proof is easily completed by induction.

Corollary 3. If the condition (34) is fulfilled, then every vector $\left(x_{1}, \ldots, x_{n}\right) \in L^{n}$ satisfying

$$
F\left(x_{1}, \ldots, x_{k-1}, 0,0, \ldots, 0\right) \leqq x_{k} \leqq G\left(x_{1}, \ldots, x_{k-1}, 1,1, \ldots, 1\right)
$$

for $k=1,2, \ldots, n$, is a solution of (27).

Theorem 4 can be specialized in the case when the lattice $L$ is biresiduated, i.e. when it is residuated with respect to the meet and join operations. In other words, this means that for every two elements $a, b \in L$, there exists an element $a: b \in L$ and an element $a: b \in L$ such that $b x \leqq a$ if and only if $x \leqq a: b$, and $a \leqq b \cup x$ if and only if $a:: b \leqq x$. Boolean algebras and totally ordered sets with 0 and 1 are examples of biresiduated lattices; the Cartesian product $L_{1} \times L_{2}$ of two biresiduated lattices $L_{1}$ and $L_{2}$ is also biresiduated.

Theorem 5. Assume the lattice $L$ is biresiduated. If the condition (34) is fulfilled, then the solutions of the inequality (27) are given by

$$
\begin{aligned}
F\left(x_{1}, \ldots, x_{k-1}, 0,0, \ldots, 0\right):: G\left(x_{1}, \ldots, x_{k-1}, 0,1, \ldots, 1\right) \leqq x_{k} \\
\leqq G\left(x_{1}, \ldots, x_{k-1}, 1,1, \ldots, 1\right): F\left(x_{1}, \ldots, x_{k-1}, 1,0, \ldots, 0\right),
\end{aligned}
$$

for $k=1,2, \ldots, n$.

Proof. The result follows immediately from Theorem 4.

Theorem 5 generalizes a result proved by M. Gotō [2] for the two-element Boolean algebra and by the present author [5], [6] for arbitrary Boolean algebras; see also V. N. Grebenščikov [3].

The next theorem refers again to the general case of an arbitrary distributive lattice; it generalizes a theorem on Boolean functions which goes back to A. N. Whitehead [7].

Theorem 6. Every lattice function $f: L^{n} \rightarrow L$ maps $L^{n}$ onto the interval

$$
[F(0, \ldots, 0), F(1, \ldots, 1)] \text {. }
$$


Proof (essentially given in [1]). Let $c$ be an element satisfying

$$
F(0, \ldots, 0) \leqq c \leqq G(1, \ldots, 1) ;
$$

we have to prove that the equation $f\left(x_{1}, \ldots, x_{n}\right)=c$, which is equivalent to the inequality

$$
f\left(x_{1}, \ldots, x_{n}\right) \cup c \leqq f\left(x_{1}, \ldots, x_{n}\right) c,
$$

is solvable. Taking into account Theorem 3, we see that the condition (34) for the inequality $(40)$ becomes $F(0, \ldots, 0) \cup c \leqq F(1, \ldots, 1) c$ and it is satisfied, because (39) implies that $F(0, \ldots, 0) \cup c=c=F(1, \ldots, 1) c$.

Conversely, it follows from Theorem 1 that

$$
F(0, \ldots, 0) \leqq f\left(x_{1}, \ldots, x_{n}\right) \leqq \bigcup_{i_{1}}, \ldots, i_{n} F\left(i_{1}, \ldots, i_{n}\right)=F(1, \ldots, 1) .
$$

Assume now that the lattice $L$ has least and greatest elements and denote them by 0 and 1 , respectively. Reasoning as in the proof of Theorem $D$ in [1], we see that the coefficients $F\left(i_{1}, \ldots, i_{n}\right)$ occurring in the canonical form of a lattice function $f\left(x_{1}, \ldots, x_{n}\right)$ are simply $F\left(i_{1}, \ldots, i_{n}\right)=f\left(i_{1}, \ldots, i_{n}\right)$. Hence the theorems proved in Sections 1-2 of [1] are particular cases of our results.

\section{REFERENCES}

(1) R. L. Goodstein, The Solution of Equations in a Lattice, Proc. Roy. Soc. Edinburgh, Sect. A, 67 (1966/67), 231-242.

(2) M. Gotō, On the General Solution of a Logical Algebraic Equation with Many Unknowns (in Japanese), Bull. Electr. Lab. 20 (1956), 81-87.

(3) V. N. Grebenščrkov, Coalitions of Systems of Equations of a Boolean Algebra and their Solution (in Russian), Dokl. Akad. Nauk SSSR, 141 (1961), 1317-1319.

(4) L. LöWENHEIM, Uber die Auflösung von Gleichungen im logischen Gebietkalkul. Math. Ann. 68 (1910), 169-207.

(5) S. Rudeanu, Boolean Equations and their Applications to the Study of Bridge Circuits. II. (in Romanian). Com. Acad. R.P. Romîne 12 (1961), 611-618.

(6) S. RudeANu, Remarks on Motinori Gotō's Papers on Boolean Equations, Rev. Roumaine Math. Pures Appl. 10 (1965), 311-317.

(7) A. N. WhITEHEAD, A Treatise on Universal Algebra, With Applications (Cambridge, 1898).

INSTITUTE OF MATHEMATICS,

EMINESCU STR. 47, Bucharest 9, Rumania 